\title{
Evaluation of WarmStart Colorimetric Loop-Mediated Isothermal Amplification Assay for Diagnosis of Malaria
}

\author{
Meng Yee Lai, ${ }^{1}$ Choo Huck Ooi, ${ }^{2}$ Joel Judson Jaimin, ${ }^{3}$ and Yee Ling Lau ${ }^{1 *}$ \\ ${ }^{1}$ Department of Parasitology, Faculty of Medicine, University of Malaya, Kuala Lumpur, Malaysia; ${ }^{2}$ Sarawak State Health Department, Kuching, \\ Malaysia; ${ }^{3}$ Health Department of Sabah State, Kota Kinabalu, Malaysia
}

\begin{abstract}
The incidence of zoonotic malaria, Plasmodium knowlesi, infection is increasing and now is the major cause of malaria in Malaysia. Here, we describe a WarmStart colorimetric loop-mediated isothermal amplification (LAMP) assay for the detection of Plasmodium spp. The detection limit for this assay was $10 \mathrm{copies} / \mu \mathrm{L}$ for $P$ knowlesi and Plasmodium ovale and $1 \mathrm{copy} / \mu \mathrm{L}$ for Plasmodium falciparum, Plasmodium vivax, and Plasmodium malariae. To test clinical sensitivity and specificity, 100 microscopy-positive and 20 malaria-negative samples were used. The WarmStart colorimetric LAMP was $98 \%$ sensitive and $100 \%$ specific. Amplification products were visible for direct observation, thereby eliminating the need for post-amplification processing steps. Therefore, WarmStart colorimetric LAMP is suitable for use in resourcelimited settings.
\end{abstract}

\section{INTRODUCTION}

Malaria continues to be one of the important parasitic infections in Malaysia despite massive investments of management time and money. Most of the malaria cases are caused by Plasmodium knowlesi infection, especially in Malaysian Borneo. Microscopic examination of blood smears is referred to as the gold standard, although this test is less sensitive than molecular diagnostic methods. ${ }^{1}$ However, it requires experienced and well-trained personnel to distinguish parasites accurately. To overcome the deficiency in microscopy examination, molecular methods are widely used. PCR is highly specific and sensitive; however, it requires expensive equipment and polymerase chain reaction $(P C R)$ reagents that are challenging to acquire in low-resource settings.

\section{MATERIALS AND METHODS}

In an attempt to mitigate this weakness, loop-mediated isothermal amplification (LAMP) has been developed. ${ }^{2}$ Loopmediated isothermal amplification is a simple and rapid detection method that can synthesize a large amount of DNA within 60 minutes without the need for complicated and expensive equipment.

The WarmStart colorimetric LAMP reagent is designed to allow for the visual detection of LAMP products by direct observation. The inclusion of $\mathrm{pH}$-sensitive dye phenol red in the master mix fosters color changes from pink to yellow color if a positive reaction occurs. On positive amplification, protons are produced because of the drop in $\mathrm{pH}$ in the solution, resulting in a color change. The whole LAMP reaction can be processed using simple equipment, such as a heating block.

In this study, a total of 100 blood samples collected in ethylenediaminetetraacetic acid blood tubes $(n=85)$ and blood spots $(n=15)$ were obtained from Sabah and Sarawak State Health Department. The samples were collected from the years 2017 to 2019. DNA was extracted from blood spots and whole blood using the DNeasy ${ }^{\circledR}$ Blood and Tissue Kit

*Address correspondence to Yee-Ling Lau, Department of Parasitology, Faculty of Medicine, Jalan Universiti, Kuala Lumpur 50603, Malaysia. E-mail: lauyeeling@um.edu.my
(Qiagen, Hilden, Germany) according to the manufacturer's protocol. The samples were previously examined under a microscope before DNA extraction. Ethical approval was obtained from the Medical Research and Ethics Committee of the Ministry of Health Malaysia (NMRR-15-672-23975) and the Medical Ethics Committee of the University of Malaya Medical Centre (MEC Ref. No. 817.1). Species identification was carried out by nested PCR targeting the Plasmodium small subunit ribosomal RNA gene.,4

The WarmStart colorimetric LAMP assay was conducted using the primers targeted on the 18S rRNA gene that has been described by Lau et al. ${ }^{5}$ The LAMP assay was performed in a $25-\mu \mathrm{L}$ reaction mixture that consisted of $12.5 \mu \mathrm{L}$ of WarmStart colorimetric LAMP $2 \times$ master mix (NEB, Ipswich, United Kingdom) at $65^{\circ} \mathrm{C}$ in a Loopamp Realtime Turbidimeter (LA 500; Eiken Chemical Co., Ltd., Tokyo, Japan). The reaction was performed in separate reaction tubes for each Plasmodium species ( $P$. knowlesi, Plasmodium ovale, Plasmodium vivax, Plasmodium falciparum, and Plasmodium malariae).

To test the analytical sensitivity of the WarmStart colorimetric LAMP primer, fragments amplified by outer LAMP primers for each of the species were cloned into pGEM-T vector (Promega, Madison, WI). The recombinant plasmids were extracted using the Qiagen Spin Miniprep kit (Qiagen, Hilden, Germany) and sent for sequencing to validate their identity. To determine the limit of detection of the colorimetric LAMP primer, the copy number of the plasmid was calculated based on the following formula: number of copies = (amount of plasmid [ng] $\left.\times 6.022 \times 10^{23}\right) /\left(\right.$ length of plasmid [bp] $\times 1 \times 10^{9} \times$ $650)$. A 10 -fold serial dilution of the plasmid $\left(10^{6}\right.$ copies to one copy) was performed with sterile distilled water. To detect the sensitivity of the WarmStart colorimetric LAMP assay, $1 \mu \mathrm{L}$ of each of the diluted DNA plasmids was used as the template. In the work presented here, the detection limit for $P$. knowlesi and $P$. ovale was 10 copies, whereas the detection limit for $P$. vivax, $P$. falciparum, and $P$. malariae was as low as 1 copy. Loopmediated isothermal amplification was repeated twice for each of the dilutions. The limit of detection of the WarmStart colorimetric LAMP assay was lower than that of nested PCR, which had a detection limit of 100 copies for $P$. knowlesi and $P$. ovale and 10 copies for $P$. vivax and $P$. malariae,$^{5}$ well less than the threshold of detection for microscopy with 50 parasites $/ \mu L{ }^{6}$ 
TABLE 1

Overall sensitivity and specificity of the nested PCR and LAMP assay

\begin{tabular}{|c|c|c|c|c|c|c|c|c|}
\hline Method & P. knowlesi & P. ovale & P. vivax & P. falciparum & P. malariae & $\begin{array}{l}\text { Healthy } \\
\text { donor }\end{array}$ & $\begin{array}{c}\text { Overall sensitivity, } \\
\%(95 \% \mathrm{Cl})\end{array}$ & $\begin{array}{c}\text { Overall specificity, } \\
\%(95 \% \mathrm{Cl})\end{array}$ \\
\hline \multicolumn{9}{|l|}{ Microscopy } \\
\hline Positive & 42 & $4 \dagger$ & $21^{*}$ & 10 & $23 \dagger$ & 0 & - & - \\
\hline Negative & 0 & 0 & 0 & 0 & 0 & 20 & - & - \\
\hline \multicolumn{9}{|l|}{ Nested PCR } \\
\hline Positive & $37+1^{*}$ & 3 & 19 & $10+8+$ & 16 & 0 & $94(87.4-97.8)$ & $100(83.2-100)$ \\
\hline $\begin{array}{l}\text { Negative } \\
\text { LAMP }\end{array}$ & 5 & 0 & 1 & 0 & 0 & 20 & - & - \\
\hline Positive & $41+1^{*}$ & 3 & 19 & $10+8 \dagger$ & 16 & 0 & 98 (92.9-99.8) & $100(83.2-100)$ \\
\hline Negative & 1 & 0 & 1 & 0 & 0 & 20 & - & - \\
\hline
\end{tabular}

The specificity of the WarmStart colorimetric LAMP assay was tested using DNA templates from non-Plasmodium parasites, including one Toxoplasma gondii, two Sarcocystis spp., and five Brugia spp. samples. The results show that the specificity was $100 \%$.

The clinical sensitivity and specificity of nested PCR and the WarmStart colorimetric LAMP assay were evaluated using 100 blood samples (parasitemia ranged from $0.1 \%$ to $3.6 \%$ ) and 20 healthy donors, with microscopy as the reference method. Sensitivity was calculated as follows: (number of true positives)/(number of true positives + number of false negatives), and specificity was calculated as follows: (number of true negatives)/(number of true negatives + number of false positives). The WarmStart colorimetric LAMP assay did not detect any of the negative DNA samples. It was $98 \%$ sensitive and $100 \%$ specific (Table 1 ).

\section{RESULTS}

Loop-mediated isothermal amplification sensitivity and specificity were the same for DNA isolated from blood or dried blood spots. Samples could be collected in the form of blood spots on a filter paper or in a blood tube. In the present study, seven samples were identified as Plasmodium malaraie and one sample as $P$. ovale based on microscopy; however, these samples were detected as $P$. falciparum via both WarmStart colorimetric LAMP and nested PCR. Apart from this, one $P$. vivax-positive sample based on microscopy was detected as $P$. knowlesi via both colorimetric LAMP and nested PCR. Of 100 samples, two samples were identified as $P$. knowlesi and $P$. vivax, respectively, based on microscopy but negative via both WarmStart colorimetric LAMP and nested PCR.

TABLE 2

Sensitivity and specificity of the nested PCR and LAMP assay for each of the species

\begin{tabular}{llcc}
\hline Plasmodium spp. & \multicolumn{1}{c}{ Method } & Sensitivity, \% (95\% Cl) & Specificity, \% (95\% Cl) \\
\hline Plasmodium & Nested PCR & $88.1(74.4-96)$ & $98.7(93.1-99.9)$ \\
knowlesi & LAMP & $97.7(87.4-99.9)$ & $98.7(93.1-99.9)$ \\
Plasmodium & Nested PCR & $75(19.4-99.4)$ & $100(96.9-100)$ \\
ovale & LAMP & $75(19.4-99.4)$ & $100(96.9-100)$ \\
Plasmodium & Nested PCR & $90.5(69.6-98.8)$ & $100(96.3-100)$ \\
vivax & LAMP & $90.5(69.6-98.8)$ & $100(96.3-100)$ \\
Plasmodium & Nested PCR & $100(69.2-100)$ & $92.7(86.2-96.8)$ \\
falciparum & LAMP & $100(69.2-100)$ & $92.7(86.2-96.8)$ \\
Plasmodium & Nested PCR & $69.6(47.1-86.8)$ & $100(69.2-100)$ \\
malariae & LAMP & $69.6(47.1-86.8)$ & $100(96.3-100)$ \\
\hline LAMP = loop-mediated isothermal amplification. &
\end{tabular}

Compared with microscopy, nested PCR revealed 94\% sensitivity and $100 \%$ specificity. The WarmStart colorimetric LAMP assay exhibited higher sensitivity (98\%) than nested PCR (Table 1). Furthermore, the WarmStart colorimetric LAMP assay managed to detect 98 samples (42 $P$. knowlesi, three $P$. ovale, $19 P$. vivax, $18 P$. falciparum, and $16 P$. malariae), whereas nested PCR only detected 94 positive samples (38 P. knowlesi, three P. ovale, 19P. vivax, 18 P. falciparum, and 16 $P$. malariae). Four samples were identified as $P$. knowlesi under microscopy but were negative for nested PCR. However, the WarmStart colorimetric LAMP assay managed to identify these four samples as $P$. knowlesi. This shows that the WarmStart colorimetric LAMP assay was more sensitive over nested PCR for $P$. knowlesi. However, for other species, LAMP and nested PCR showed similar results for sensitivity and specificity (Table 2).

\section{DISCUSSION}

WarmStart colorimetric LAMP monitoring relies on color changes of the end product, hence eliminating laborious postamplification work, such as gel electrophoresis and ethidium bromide gel staining. In addition, the $\mathrm{pH}$ indicator dye was included in the reaction mixture beforehand during manufacturing, thereby avoiding the additional dye for end products after amplification. The cap of the tube was not opened and, subsequently, this helped reduce the airborne aerosol being released into the surrounding areas, which can contribute to contamination. In the present study, positive and negative reactions were indicated by a yellow and pink color, respectively. The color change can significantly differentiate positive and negative reactions. Additional fluorescent intercalating dyes (such as SYBR Green (Sigma-Aldrich, St. Louis, MO) and PicoGreen) along with colorimetric HNB dye (Sigma-Aldrich) being added to the reaction mix has also been widely used. However, the results were subjective to individual eyes as the color changes between the positive and negative reactions were not significant. 7,8 These two studies revealed that the color changes based on SYBR and HNB were not significantly distinguishable. To minimize the volume of the WarmStart colorimetric LAMP buffer, a different total reaction volume of $15 \mu \mathrm{L}, 20 \mu \mathrm{L}$, and $25 \mu \mathrm{L}$ was tested. By setting up a similar reaction amplification temperature and timing, the results show no difference among $15 \mu \mathrm{L}, 20 \mu \mathrm{L}$, and $25 \mu \mathrm{L}$. Thus, only $7.5 \mu \mathrm{L}$ of the $2 \times$ master mix was needed per reaction. 
Subsequently, the cost per reaction can be saved. Using similar reagents, Baek et al. ${ }^{9}$ prepared a total $10-\mu \mathrm{L}$ reaction volume for each reaction during the detection of thrombocytopenia syndrome virus. The result shows no difference with the commonly used $25-\mu \mathrm{L}$ total reaction.

There is still some room for improvement in terms of the WarmStart colorimetric LAMP. This method cannot be used to detect multiple pathogens at the same time. Further improvement of this method may allow simultaneous detection of five human Plasmodium spp. Addition of different labels, such as Cy3, Cy5, FITC, ROX, and Texas Red, to the loop primers of each species results in the amplified DNA product emitting fluorescence at different wavelengths for the respective species.

\section{CONCLUSION}

WarmStart colorimetric LAMP assay is a robust method for the detection of human Plasmodium spp. This technique requires 30 minutes to complete the whole amplification process and shows higher sensitivity than nested PCR. The results of WarmStart colorimetric LAMP can be inspected easily by direct observation without additional color dye being added to the reaction tube at the end of the amplification. The risk of contamination can be reduced as detection is accomplished within a closed system without opening the reaction tube. Therefore, the WarmStart colorimetric LAMP assay has potential for use in resource-limited settings.

Received January 2, 2020. Accepted for publication February 23, 2020.

Published online March 30, 2020.

Financial support: This study was supported by Long Term Research Grant Scheme (LRGS), LR002D-2018, from the Ministry of Education, Malaysia.

Authors' addresses: Meng Yee Lai and Yee Ling Lau, Department of Parasitology, Faculty of Medicine, University of Malaya, Kuala Lumpur, Malaysia, E-mails: mengylai11@yahoo.com and lauyeeling@ um.edu.my. Choo Huck Ooi, Sarawak State Health Department, Kuching, Malaysia, E-mail: ooi.choo.huck@gmail.com. Joel Judson Jaimin, Health Department of Sabah State, Kota Kinabalu, Malaysia, E-mail: joeljj@moh.gov.my.

\section{REFERENCES}

1. CDC, 2019. Centers for Disease Control and Prevention. Available at: https://www.cdc.gov/malaria/diagnosis_treatment/diagnostic_ tools.html/. Accessed February 18, 2020.

2. Notomi T, Okayama H, Masubuchi $H$, Yonekawa $T$, Watanabe $K$, Amino N, Hase T, 2000. Loop-mediated isothermal amplification of DNA. Nucleic Acids Res 28: E63.

3. Snounou G, Viriyakosol S, Zhu XP, Jarra W, Pinheiro L, do Rosario VE, Thaithong S, Brown KN, 1993. High sensitivity of detection of human malaria parasites by the use of nested polymerase chain reaction. Mol Biochem Parasitol 61: 315-320.

4. Imwong M, Hanchana S, Malleret B, Rénia L, Day NP, Dondorp A, Nosten F, Snounou G, White NJ, 2014. High-throughput ultra sensitive molecular techniques for quantifying low-density malaria parasitemias. J Clin Microbiol 52: 3303-3309.

5. Lau YL, Lai MY, Fong MY, Jelip J, Mahmud R, 2016. Loopmediated isothermal amplification assay for identification of five human Plasmodium species in Malaysia. Am J Trop Med Hyg 94: 336-339.

6. World Health Organization, 2015. Microscopy for the Detection, Identification and Quantification of Malaria Parasites on Stained Thick and Thin Blood Films in Research Settings (Version 1.0): Procedure: Methods Manual. Available at: https://apps.who.int/iris/handle/10665/163782/. Accessed February 18, 2020.

7. Ghosh R, Nagavardhini A, Sengupta A, Sharma M, 2015. Development of loop-mediated isothermal amplification (LAMP) assay for rapid detection of Fusarium oxysporum $f$. sp. ciceris - wilt pathogen of chickpea. BMC Res Notes 8: 40.

8. Singh R, Singh DP, Savargaonkar D, Singh OP, Bhatt RM, Valecha $\mathrm{N}, 2017$. Evaluation of SYBR green I based visual loopmediated isothermal amplification (LAMP) assay for genus and species-specific diagnosis of malaria in $P$. vivax and $P$. falciparum endemic regions. J Vector Borne Dis 54: 54-60.

9. Baek YH et al., 2018. Simple, rapid and sensitive portable molecular diagnosis of SFTS virus using reverse transcriptional loopmediated isothermal amplification (RT-LAMP). J Microbiol Biotechnol 28: 1928-1936. 